\title{
Energy and spectral efficient Doppler diversity transmissions in high-mobility systems with imperfect channel estimation
}

\author{
Weixi Zhou ${ }^{1 *}$, Jingxian $\mathrm{Wu}^{2}$ and Pingzhi Fan ${ }^{1}$
}

\begin{abstract}
This paper studies energy and spectral efficient Doppler diversity transmissions in the presence of imperfect channel state information (CSI). Fast time-varying fading in high-mobility communication systems introduces Doppler diversity that can benefit system performance. On the other hand, it is more difficult to estimate and track fast time-varying channel; thus, channel estimation errors might seriously degrade system performance in high-mobility systems. The trade-offs between channel estimation errors and Doppler diversity are studied by using two precoding schemes, a simple repetition code, and a rate 1 Doppler domain multiplexing (DDM) scheme. The repetition code can achieve the maximum Doppler diversity at the cost of a lower spectral efficiency, and the DDM scheme can achieve the energy and spectral efficient Doppler diversity transmissions. Unlike many other Doppler diversity systems that assume perfect CSI, we explicitly consider the impacts of imperfect CSI on the design and performance of both precoding schemes. Optimum and suboptimum receivers for both schemes are developed by studying the statistical properties of channel estimation errors. The analytical error probabilities of the receivers are expressed as functions of a number of system parameters, such as the maximum Doppler spread, the percentage of pilot symbols for channel estimation, the energy allocation between the pilot and data symbols, etc. The analytical and simulation results indicate that both precoding schemes can achieve the maximum Doppler diversity order through the optimization of the various parameters, even in the presence of imperfect CSI.
\end{abstract}

Keywords: Doppler diversity; Channel estimation error; High-mobility communications

\section{Introduction}

With the increasing demands of high-speed railways and aircraft communications, wireless communications in high-mobility environment have attracted considerable attentions during the past few years. Signals in highmobility systems could experience large Doppler shifts in the order of kilohertz, while most conventional wireless communication systems are designed for Doppler shifts up to a few hundred hertz. The large Doppler shifts result in fast time-varying fading, which is one of the main challenges for the design of reliable high-mobility systems. On the other hand, fast time-varying fading caused by large Doppler shifts in high-mobility systems provides

\footnotetext{
*Correspondence: zhouweixister@gmail.com

1 Institute of Mobile Communications, Southwest Jiaotong University, Chengdu, China

Full list of author information is available at the end of the article
}

Doppler diversity, which can be used to benefit system performance.

Several methods are proposed to exploit the Doppler diversity gain inherent in fast time-varying fading [1-3], and they provide efficient countermeasures against fading. Most existing works on Doppler diversity assume that perfect channel state information (CSI) can be obtained at the receiver. However, in high-mobility systems, it is a non-trivial task to estimate and track the fast time-varying fading with high precision and credibility. Channel estimation errors are usually inevitable and might have significant impacts on system performance when the Doppler frequency is high. The performance of precoded orthogonal frequency division multiplexing (OFDM) systems with channel estimation error is studied in [4]. It is shown in [5] that for a single-input multiple-output (SIMO) system with identically and independently distributed (i.i.d.) fading, the conventional maximal ratio combining (MRC)

\section{量 Springer}

(c) 2015 Zhou et al.: licensee Springer. This is an Open Access article distributed under the terms of the Creative Commons Attribution License (http://creativecommons.org/licenses/by/4.0), which permits unrestricted use, distribution, and reproduction in any medium, provided the original work is properly credited. 
receiver is no longer optimum with imperfect CSI. The results of [4] and [5] cannot be applied to high-mobility systems because they both assume the systems experience quasi-static fading channels. The optimum designs of high-mobility systems in the presence of imperfect CSI are studied in $[6,7]$ and $[8]$ in terms of different design metrics, such as the bit error rate (BER), symbol error rate (SER), or spectral efficiency. However, Doppler diversity is not considered in these works.

In this paper, we study the energy and spectral efficient designs of Doppler diversity transmissions in highmobility systems with imperfect CSI. The design aims at achieving a balanced trade-off between Doppler diversity gain and channel estimation errors by considering a large number of system parameters, such as the percentage of pilot symbols among the total transmitted symbols, the energy allocation between pilot and data symbols, and the maximum Doppler shifts. Two precoding schemes are employed to achieve Doppler diversity: a simple repetition code, and a rate 1 Doppler domain multiplexing (DDM) code [3]. The repetition precoder can achieve the full Doppler diversity at the cost of a lower spectral efficiency, and the rate 1 DDM precoder can achieve energy and spectral efficient Doppler diversity transmissions. By analyzing the statistical properties of channel estimation errors, we develop the optimum and sub-optimum receivers for both precoding schemes. The main contributions of this paper are summarized as follows.

- The Doppler diversity transceivers in this paper are developed by explicitly utilizing the properties of imperfect CSI, whereas most existing works on Doppler diversity assume perfect CSI. For example, the DDM scheme is originally proposed in [3] for systems with perfect CSI, and its performance degrades significantly with imperfect CSI, given the fact that conventional receivers are no longer optimum with imperfect CSI. We address this problem by developing new transceiver structures that explicitly consider the impact of channel estimation errors.

- The impacts of imperfect CSI on system performance are identified by developing the theoretical error probabilities of the new transceivers. The new analytical results are expressed as functions of the second-order statistics of the channel estimation errors, and they reveal the trade-off between Doppler diversity and channel estimation errors.

- The new transceiver structure along with the theoretical error probabilities can be used to guide the development of practical Doppler diversity systems. With the analytical and simulation results, the various system parameters that yield the optimum trade-off between channel estimation errors and Doppler diversity are identified.

The rest of this paper is organized as follows. Section 2 introduces the system model and the two precoding schemes. Section 3 shows the channel estimation. The trade-off between Doppler diversity and channel estimation errors is studied in Section 4 with the repetition code. Section 5 presents the receiver design and performance analysis of the rate 1 DDM scheme. Numerical and simulation results are presented in Section 6, and Section 7 concludes the paper.

\section{System model}

The system model is presented in this section. At the transmitter, the modulated data symbols are precoded to achieve Doppler diversity transmissions. Pilot symbols are inserted among the precoded data systems to track and estimate the fast time-varying fading channel.

\subsection{Precoding}

The data symbols to be transmitted are divided into slots. Denote the modulated data symbols in one slot as $\mathbf{s}=$ $\left[s_{1}, \cdots, s_{N_{s}}\right]^{T} \in \mathcal{S}^{N_{s}}$, where $\mathcal{S}$ is the modulation alphabet set, $\mathbf{A}^{T}$ is the matrix transpose operation, and $N_{s}$ is the number of data symbols in one slot. To achieve the maximum Doppler diversity, precoding is employed to spread out the data symbols in the time domain before transmission. The precoded data vector can be represented by

$$
\mathbf{c}=\Theta \mathbf{s}
$$

where $\Theta \in \mathcal{C}^{N_{c} \times N_{s}}$ is a precoding matrix with $\mathcal{C}$ being the set of complex numbers, and $N_{c} \geq N_{s}$ is the length of the codeword. Define the $k$-th precoded data symbol of $\mathbf{c}=\left[c_{1}, \cdots, c_{N_{c}}\right]^{T}$ as $c_{k}$, where $k=1, \cdots, N_{c}$. The coding rate is $\rho=\frac{N_{s}}{N_{c}}$ and the precoding matrix satisfies trace $\left(\Theta \Theta^{H}\right)=N_{s}$, with $\mathbf{A}^{H}$ being the matrix Hermitian operation. Two precoding schemes are considered in this paper.

The first precoding scheme is a simple rate $\frac{1}{N_{c}}$ repetition code with $N_{s}=1$ and $\Theta=\frac{1}{\sqrt{N_{c}}} \mathbf{1}_{N_{c}}$, where $\mathbf{1}_{N_{c}}$ is a length- $N_{c}$ all-one vector. Since the repetition code spreads one data symbol over the entire slot, it is guaranteed to achieve the maximum Doppler diversity at the cost of a low spectral efficiency. The repetition code provides the best possible performance in terms of Doppler diversity gain, and its performance can serve as a lower bound for practical precoding schemes [9].

The second precoding scheme is the rate 1 DDM scheme originally proposed in [3]. It can achieve the maximum Doppler diversity without sacrificing spectral efficiency, at the cost of mutual interference among the 
data streams. The precoding matrix is $\Theta=\mathbf{F}_{N_{c}}^{H}$, where $N_{c}=N_{s}$ and $\mathbf{F}_{N_{c}} \in \mathcal{C}^{N_{c} \times N_{c}}$ is the normalized discrete Fourier transform (DFT) matrix with the $(n, k)$-th element being $\frac{1}{\sqrt{N_{c}}} e^{-j 2 \pi(n-1)(k-1) / N_{c}}$. With DDM, the codeword can be alternatively expressed as

$$
\mathbf{c}=\sum_{n=1}^{N_{c}} \theta_{n} s_{n},
$$

where $\theta_{n}$ is the $n$-th column of $\Theta$. With such a precoding scheme, the $n$-th data symbol is spread out over the entire slot as $\theta_{n} s_{n}$, such that the maximum Doppler diversity can be achieved. However, the orthogonality between the columns of the precoding matrix will be destroyed by the fast time-varying fading channel. Therefore, there will be mutual interferences among the data symbols at the receiver. The interference can be partly removed by means of Doppler domain equalization. It should be noted that perfect CSI at the receiver is assumed by [3], and this assumption is usually not true for high-mobility systems.

\subsection{Pilot-assisted transmission}

After precoding, $N_{p}$ equally-spaced pilot symbols are inserted in each slot to assist channel estimation at the receiver. The number of precoded data symbols $N_{c}$ and pilot symbols $N_{p}$ are chosen as $N_{c}=N_{p}(K-1)$, such that there are $K-1$ precoded data symbols between a pair of adjacent pilot symbols. The $k$-th pilot symbol is denoted as $x_{i_{k}}=p_{k}$, where $i_{k}=k K$ is the index of the $k$-th pilot symbol, for $k=1, \ldots, N_{p}$. Similarly, the $k$-th data symbol is denoted as $x_{n_{k}}=c_{k}$, where $n_{k}=k+\left\lfloor\frac{k-1}{K-1}\right\rfloor$ is the index of the $k$-th coded data symbol, for $k=1, \cdots, N_{c}$, where $\lfloor a\rfloor$ is the largest integer not larger than $a$. Define $\mathbf{x}_{p}=\left[x_{i_{1}}, \cdots, x_{i_{N_{p}}}\right]^{T}$ and $\mathbf{x}_{d}=\left[x_{n_{1}}, \cdots, x_{n_{N_{c}}}\right]^{T}$ as the pilot and coded data vectors, respectively. Pilot and data symbols can be from different modulation alphabet sets. Without loss of generality, pilot symbols are assumed to be constant amplitude symbols, i.e., $\left|p_{k}\right|^{2}=1$, which is not necessarily the case for data symbols. With such a slot structure, the pilot percentage can be defined as $\delta=\frac{N_{p}}{N}=$ $\frac{1}{K}$, with $N=N_{p}+N_{c}$.

Due to the different roles of channel estimation and data transmission, different amounts of energy are allocated to the pilot and coded data symbols. Denote the energy for each pilot and coded data symbol as $E_{p}$ and $E_{c}$, respectively. The total energy in one slot is thus $E_{0}=$ $E_{p} N_{p}+E_{c} N_{c}$. The energy per uncoded information bit can be calculated as $E_{b}=\frac{E_{p} N_{p}+E_{c} N_{c}}{N_{s} \log _{2} M}$, where $M=|\mathcal{S}|$ is the cardinality of the modulation constellation set. Define an energy allocation factor $\eta=\frac{E_{p} N_{p}}{E_{0}} \in(0,1)$ as the fraction of the total energy per slot allocated to pilot symbols, $E_{p}$ and $E_{c}$ can be expressed by

$$
\begin{aligned}
& E_{p}=\frac{\eta E_{0}}{N_{p}}=\eta\left(\frac{1}{\delta}-1\right) \rho E_{b} \log _{2} M, \\
& E_{c}=\frac{(1-\eta) E_{0}}{N_{c}}=(1-\eta) \rho E_{b} \log _{2} M,
\end{aligned}
$$

where $\rho$ is the precoding code rate. Under a fixed $E_{b}, \eta$, and $\rho$, increasing the pilot percentage $\delta$ will decrease the energy for each pilot symbol; however, it will not affect the energy per coded data symbol.

The pilot and coded data symbols are transmitted over the time-varying fading channel. The pilot symbols observed at the receiver can be represented by

$$
\mathbf{y}_{p}=\sqrt{E_{p}} \mathbf{X}_{p} \mathbf{h}_{p}+\mathbf{z}_{p}
$$

where $\mathbf{y}_{p}=\left[y\left(i_{1}\right), \cdots, y\left(i_{N_{p}}\right)\right]^{T} \in \mathcal{C}^{N_{p} \times 1}$ and $\mathbf{z}_{p}=\left[z\left(i_{1}\right), \cdots\right.$, $\left.z\left(i_{N_{p}}\right)\right]^{T} \in \mathcal{C}^{N_{p} \times 1}$ are the received pilot vector and additive white Gaussian noise (AWGN) vector, respectively, and $\mathbf{X}_{p}=\operatorname{diag}\left(\mathbf{x}_{p}\right)$ is a diagonal matrix with the main diagonal being $\mathbf{x}_{p}$, and $\mathbf{h}_{p}=\left[h\left(i_{1}\right), \ldots, h\left(i_{N_{p}}\right)\right]^{T} \in \mathcal{C}^{N_{p} \times 1}$ is the discrete-time channel fading vector. The AWGN vector is a zero-mean symmetric complex Gaussian random vector (CGRV) with covariance matrix $N_{0} \mathbf{I}_{N_{p}}$, where $\mathbf{I}_{N}$ is a size $N$ identity matrix.

Similarly, the data symbols observed at the receiver can be denoted as,

$$
\mathbf{y}_{d}=\sqrt{E_{c}} \mathbf{x}_{d} \mathbf{h}_{d}+\mathbf{z}_{d}
$$

where $\mathbf{y}_{d}=\left[y\left(n_{1}\right), \cdots, y\left(n_{N_{c}}\right)\right]^{T} \in \mathcal{C}^{N_{c} \times 1}$ and $\mathbf{z}_{d}=\left[z\left(n_{1}\right), \cdots\right.$, $\left.z\left(n_{N_{c}}\right)\right]^{T} \in \mathcal{C}^{N_{c} \times 1}$ are received coded data symbols and AWGN, respectively, $\mathbf{X}_{d}=\operatorname{diag}\left(\mathbf{x}_{d}\right)$ is a diagonal matrix with the precoded data vector $\mathbf{x}_{d}$ on its main diagonal, and $\mathbf{h}_{d}=\left[h\left(n_{1}\right), \ldots, h\left(n_{N_{c}}\right)\right]^{T} \in \mathcal{C}^{N_{c} \times 1}$.

\section{Channel estimation}

The channel is assumed to experience wide-sense stationary uncorrelated scattering, and $h(n)$ is a zero-mean symmetric complex Gaussian random process with the covariance function

$$
\phi(m-n)=\mathbb{E}\left[h(m) h^{*}(n)\right]=J_{0}\left(2 \pi f_{\mathrm{D}}|m-n| T_{s}\right),
$$

where $a^{*}$ denotes complex conjugate, $f_{\mathrm{D}}$ is the maximum Doppler spread of the fading channel, $T_{s}$ is the symbol period, and $J_{0}(x)$ is the zero-order Bessel function of the first kind.

In fast time-varying fading, the channel coefficients vary from symbol to symbol by following the time correlation in (7). As a result, the channel coefficients of the pilot symbols, $\mathbf{h}_{p}$, are different from the channel coefficients of the data symbols, $\mathbf{h}_{d}$. 
In order to track the fast time variation of the fading coefficients, the channel estimation can be performed in two steps. In the first step, the receiver obtains an estimate of the channel coefficients at pilot locations as

$$
\hat{\mathbf{h}}_{p}=\mathbf{W}_{p}^{H} \mathbf{y}_{p},
$$

where $\mathbf{W}_{p} \in \mathcal{C}^{N_{p} \times N_{p}}$ is the minimum mean squared error (MMSE) matrix designed to minimize the mean squared error (MSE) $\frac{1}{N_{p}} \mathbb{E}\left(\left\|\hat{\mathbf{h}}_{p}-\mathbf{h}_{p}\right\|^{2}\right)$. In the second step, the channel coefficients at data locations can be estimated by performing time domain interpolation over $\hat{\mathbf{h}}_{p}$ as

$$
\hat{\mathbf{h}}_{d}=\mathbf{W}_{d}^{H} \hat{\mathbf{h}}_{p},
$$

where $\mathbf{W}_{d} \in \mathcal{C}^{N_{c} \times N_{p}}$ is the MMSE matrix to minimize $\frac{1}{N_{c}} \mathbb{E}\left(\left\|\hat{\mathbf{h}}_{d}-\mathbf{h}_{d}\right\|^{2}\right)$.

It is shown in [10] and [11] that the above two-step MMSE is equivalent to a single-step MMSE as

$$
\hat{\mathbf{h}}_{d}=\mathbf{W}_{d}^{H} \hat{\mathbf{h}}_{p}=\mathbf{W}_{d}^{H} \mathbf{W}_{p}^{H} \mathbf{y}_{p}=\mathbf{W}^{H} \mathbf{y}_{p},
$$

where $\mathbf{W}=\mathbf{W}_{p} \mathbf{W}_{d} \in \mathcal{C}^{N_{c} \times N_{p}}$ is the MMSE matrix that can minimize $\frac{1}{N_{c}} \mathbb{E}\left(\left\|\hat{\mathbf{h}}_{d}-\mathbf{h}_{d}\right\|^{2}\right)$. In (10), the received pilot symbols are used to estimate the channel coefficients of data symbols through time interpolation; thus, the fast time-variation of the fading coefficients can be accurately tracked.

Based on the orthogonal principal, $\mathbb{E}\left[\left(\hat{\mathbf{h}}_{d}-\mathbf{h}_{d}\right) \mathbf{y}_{p}^{H}\right]=$ 0, the MMSE estimation matrix can be solved as

$$
\mathbf{W}^{H}=\sqrt{E_{p}} \mathbf{R}_{d p} \mathbf{X}_{p}^{H}\left(E_{p} \mathbf{X}_{p} \mathbf{R}_{p p} \mathbf{X}_{p}^{H}+\sigma_{z}^{2} \mathbf{I}_{N_{p}}\right)^{-1},
$$

where $\mathbf{A}^{-1}$ is the matrix inverse operation, $\mathbf{R}_{d p}=$ $\mathbb{E}\left[\mathbf{h}_{d} \mathbf{h}_{p}^{H}\right] \in \mathcal{R}^{N_{c} \times N_{p}}$ and $\mathbf{R}_{p p}=\mathbb{E}\left[\mathbf{h}_{p} \mathbf{h}_{p}^{H}\right] \in \mathcal{C}^{N_{p} \times N_{p}}$ with their elements defined in (7).

Define the error vector $\mathbf{e}_{d}=\mathbf{h}_{d}-\hat{\mathbf{h}}_{d}$. The correlation of the error vector, $\mathbf{R}_{e e}=\mathbb{E}\left[\mathbf{e}_{d} \mathbf{e}_{d}^{H}\right] \in \mathcal{R}^{N_{c} \times N_{c}}$, can be calculated as

$$
\mathbf{R}_{e e}=\mathbf{R}_{d d}-\mathbf{R}_{d p}\left(\mathbf{R}_{p p}+\frac{1}{\gamma_{p}} \mathbf{I}_{N_{p}}\right)^{-1} \mathbf{R}_{d p}^{H} .
$$

where $\gamma_{p}=\frac{E_{p}}{\sigma_{z}^{2}}$ is the signal-to-noise ratio (SNR) of the pilot symbols, $\mathbf{R}_{d d}=\mathbb{E}\left[\mathbf{h}_{d} \mathbf{h}_{d}^{H}\right] \in \mathcal{R}^{N_{c} \times N_{c}}$, and $\mathbf{X}_{p} \mathbf{X}_{p}^{H}=$ $\mathbf{I}_{N_{p}}$ is used in the derivation of the above equation.

For the design and analysis of the diversity receiver in the presence of imperfect CSI, it is necessary to obtain the statistical properties of $\hat{\mathbf{h}}_{d}$ by considering the effects of channel estimation errors because the receiver performs detection based on the knowledge of the estimated channel coefficients $\hat{\mathbf{h}}_{d}$.
Given the pilot symbols, the vector $\mathbf{y}_{p}$ is zero-mean Gaussian distributed with covariance matrix $\mathbb{E}\left[\mathbf{y}_{p} \mathbf{y}_{p}^{H}\right]=$ $E_{p} \mathbf{X}_{p} \mathbf{R}_{p p} \mathbf{X}_{p}^{H}+\sigma_{z}^{2} \mathbf{I}_{N_{p}}$. From (10), the estimated channel coefficient vector is a linear transformation of a zero-mean Gaussian vector. Therefore, $\hat{\mathbf{h}}_{d}$ is zero-mean Gaussian distributed with the covariance matrix

$$
\mathbf{R}_{\hat{d} \hat{d}}=\mathbf{R}_{d p}\left(\mathbf{R}_{p p}+\frac{1}{\gamma_{p}} \mathbf{I}_{N_{p}}\right)^{-1} \mathbf{R}_{d p}^{H}=\mathbf{R}_{d d}-\mathbf{R}_{e e} .
$$

To gain further insights, define the estimation MSE as $\sigma_{e, N_{c}}^{2}=\frac{1}{N_{c}} \operatorname{trace}\left(\mathbf{R}_{e e}\right)$. When $N_{c}$ goes to infinity while keeping a finite $\delta$, the asymptotic MSE $\sigma_{e}^{2}=$ $\lim _{N_{c} \rightarrow \infty} \sigma_{e, N_{c}}^{2}$ is [12, Proposition 1],

$$
\sigma_{e}^{2}=1-\frac{8 v \gamma_{b} \arctan \left(\sqrt{\frac{2 v \gamma_{b}-\frac{\omega_{\mathrm{D}}}{\delta}}{2 v \gamma_{b}+\frac{\omega_{\mathrm{D}}}{\delta}}}\right)}{\pi \sqrt{\left(2 v \gamma_{b}\right)^{2}-\left(\frac{\omega_{\mathrm{D}}}{\delta}\right)^{2}}}, \text { for } \delta \geq 2 f_{\mathrm{D}} T_{s} \text {, }
$$

where $\omega_{\mathrm{D}}=2 \pi f_{\mathrm{D}} T_{s}, \gamma_{b}=E_{b} / N_{0}$ is the equivalent SNR of the uncoded bit, and $v=\eta\left(\frac{1}{\delta}-1\right) \rho \log _{2} M$.

\section{Trade-off between channel estimation errors and Doppler diversity}

The trade-off between channel estimation errors and the maximum Doppler diversity gain is studied in this section with the help of the simple repetition code.

\subsection{Optimum diversity receiver with imperfect CSI}

With the repetition precoder, the received data vector in (6) can be simplified to

$$
\mathbf{y}_{d}=\sqrt{\frac{E_{c}}{N_{c}}} \mathbf{h}_{d} s+\mathbf{z}_{d}
$$

It should be noted that the total energy of the $N_{c}$ repeated data symbols is normalized to $E_{c}$ to ensure fair comparison with other precoding schemes and uncoded systems.

The receiver performs detection based on the received data vector $\mathbf{y}_{d}$ and the knowledge of the estimated CSI vector $\hat{\mathbf{h}}_{d}$. Since both $\mathbf{h}_{d}$ and $\hat{\mathbf{h}}_{d}$ are zero-mean Gaussian distributed, the error vector $\mathbf{e}_{d}=\mathbf{h}_{d}-$ $\hat{\mathbf{h}}_{d}$ is zero-mean Gaussian distributed. Conditioned on $\hat{\mathbf{h}}_{d}, \mathbf{h}_{d}$ is Gaussian distributed with mean $\mathbf{u}_{d \mid \hat{h}_{d}}=$ $\mathbb{E}\left[\mathbf{h}_{d} \mid \hat{\mathbf{h}}_{d}\right]=\hat{\mathbf{h}}_{d}$ and covariance matrix $\mathbf{R}_{d d \mid \hat{h}_{d}}=$ $\mathbb{E}\left[\left(\mathbf{h}_{d}-\mathbf{u}_{d \mid \hat{h}_{d}}\right)\left(\mathbf{h}_{d}-\mathbf{u}_{d \mid \hat{h}_{d}}\right)^{H} \mid \hat{\mathbf{h}}_{d}\right]=\mathbf{R}_{e e}$.

Therefore, conditioned on the transmitted data symbol $s_{m}$ and the estimated CSI vector $\hat{\mathbf{h}}_{d}$, the received data vector $\mathbf{y}_{d}$ is complex Gaussian distributed , i.e., $\mathbf{y}_{d} \mid\left(\hat{\mathbf{h}}_{d}, s_{m}\right) \sim$ 
$\mathcal{C} N\left(\mathbf{u}_{y \mid \hat{h}_{d}, s_{m}}, \mathbf{R}_{y y \mid \hat{h}_{d}, s_{m}}\right)$. The conditional mean and conditional covariance matrix are given as follows

$$
\begin{aligned}
\mathbf{u}_{y \mid \hat{h}_{d}, s_{m}} & =\sqrt{\frac{E_{c}}{N_{c}}} \hat{\mathbf{h}}_{d} s_{m}, \\
\mathbf{R}_{y y \mid \hat{h}_{d}, s_{m}} & =\frac{E_{c}}{N_{c}} \mathbf{R}_{e e}+\sigma_{z}^{2} \mathbf{I}_{N_{c}} .
\end{aligned}
$$

For system with equiprobable symbols, the maximum likelihood (ML) detection rule can minimize the detection error probability. From (16), maximizing the likelihood function, $p\left(\mathbf{y}_{d} \mid \hat{\mathbf{h}}_{d}, s_{m}\right)$, is equivalent to minimize the following cost function

$$
q\left(s_{m}\right)=\left(\mathbf{y}_{d}-\sqrt{\frac{E_{c}}{N_{c}}} \hat{\mathbf{h}}_{d} s_{m}\right)^{H} \mathbf{R}_{y y \mid \hat{h}_{d}, s_{m}}^{-1}\left(\mathbf{y}_{d}-\sqrt{\frac{E_{c}}{N_{c}}} \hat{\mathbf{h}}_{d} s_{m}\right) .
$$

It can be easily shown that

$$
\begin{aligned}
\underset{s_{m} \in \mathcal{S}}{\operatorname{argmin}} q\left(s_{m}\right) & =\underset{s_{m} \in \mathcal{S}}{\operatorname{argmin}}\left\{-2 \Re\left(\alpha \cdot s_{m}\right)\right\} \\
& =\underset{s_{m} \in \mathcal{S}}{\operatorname{argmin}}\left\{\left|\alpha-s_{m}\right|^{2}\right\},
\end{aligned}
$$

where $\mathfrak{R}$ denotes the real part operator, and $\alpha$ is the decision variable defined as

$$
\alpha=\sqrt{\frac{E_{c}}{N_{c}}} \hat{\mathbf{h}}_{d}^{H}\left(\frac{E_{c}}{N_{c}} \mathbf{R}_{e e}+\sigma_{z}^{2} \mathbf{I}_{N_{s}}\right)^{-1} \mathbf{y}_{d} .
$$

Therefore, the optimum decision rule in the presence of imperfect CSI can be written as

$$
\hat{s}=\underset{s_{m} \in \mathcal{S}}{\operatorname{argmin}}\left\{\left|\alpha-s_{m}\right|^{2}\right\}
$$

\subsection{Performance analysis}

The error performance for systems with $M$-ary phase shift keying (MPSK) modulation and operating with imperfect CSI and the optimum decision rule is derived based on the statistical properties of the estimated CSI $\hat{\mathbf{h}}_{d}$.

From [5], the conditional error probability (CEP) for systems with MPSK modulation is

$$
P\left(E \mid \hat{\mathbf{h}}_{k}\right)=\frac{1}{\pi} \int_{0}^{\pi-\frac{\pi}{M}} \exp \left[-\hat{\mathbf{h}}_{k}^{H} \mathbf{Q} \hat{\mathbf{h}}_{k} \frac{\sin ^{2}\left(\frac{\pi}{M}\right)}{\sin ^{2}(\phi)}\right] d \phi,
$$

where $\mathbf{Q}=\left(\mathbf{R}_{e e}+\frac{1}{\gamma_{c}} \mathbf{I}_{N}\right)^{-1}$.

The above CEP depends on the estimated CSI through the quantity $\beta=\hat{\mathbf{h}}_{k}^{H} \mathbf{Q} \hat{\mathbf{h}}_{k}$. Since the estimated CSI vector $\hat{\mathbf{h}}_{k}$ is zero-mean Gaussian distributed with covariance matrix $\mathbf{R}_{\hat{d} \hat{d}}$ given in (13), $\beta$ is a quadratic form of a com- plex Gaussian random vector with the moment generating function (MGF) [13]

$$
\Psi_{\beta}(t)=\mathbb{E}\left(e^{t \beta}\right)=\left[\operatorname{det}\left(\mathbf{I}_{N}-t \cdot \mathbf{R}_{\hat{d} \hat{d}} \mathbf{Q}\right)\right]^{-1},
$$

where $t$ is a dumb variable.

The MGF in (22) depends on the matrices $\mathbf{R}_{d d}$ and $\mathbf{R}_{e e}$. Perform eigenvalue decomposition of $\mathbf{R}_{e e}$ as

$$
\mathbf{R}_{e e}=\mathbf{V} \Lambda \mathbf{V}^{H}
$$

where $\mathbf{V} \in \mathcal{C}^{N_{s} \times N_{s}}$ contains the orthonormal eigenvectors of $\mathbf{R}_{e e}$, and $\Lambda=\operatorname{diag}\left[\varphi_{1}, \cdots, \varphi_{N_{c}}\right]$ is the diagonal matrix containing the corresponding eigenvalues.

Based on the Sylvester's determinant identity, the MGF in (22) can be rewritten as

$$
\Psi_{\beta}(t)=\left\{\operatorname{det}\left[\mathbf{I}_{N_{c}}-t \cdot \boldsymbol{\Omega}^{-\frac{1}{2}} \mathbf{V}^{H} \mathbf{R}_{\hat{d} \hat{d}} \mathbf{V} \boldsymbol{\Omega}^{-\frac{1}{2}}\right]\right\}^{-1},
$$

where $\boldsymbol{\Omega} \in \mathcal{R}^{N_{c} \times N_{c}}$ is a diagonal matrix with the $k$-th diagonal element being $\varphi_{k}+\frac{N_{c}}{\gamma_{c}}$.

Perform eigenvalue decomposition on the Hermitian matrix $\boldsymbol{\Upsilon}=\boldsymbol{\Omega}^{-\frac{1}{2}} \mathbf{V}^{H} \mathbf{R}_{\hat{d} \hat{d}} \mathbf{V} \boldsymbol{\Omega}^{-\frac{1}{2}}=\mathbf{U D U} \mathbf{U}^{H}$, where $\mathbf{U} \in$ $\mathcal{C}^{N_{c} \times N_{c}}$ contains the orthonormal eigenvectors of $\Upsilon$, and $\mathbf{D}=\operatorname{diag}\left\{\left[d_{1}, \cdots, d_{N_{c}}\right]\right\}$ is the diagonal matrix containing the corresponding eigenvalues. Then the MGF in (24) can be simplified to

$$
\Psi_{\beta}(t)=\prod_{n=1}^{N_{c}} \frac{1}{1-t d_{n}}
$$

From (21) and (25), the unconditional SER, $P(E)=$ $\mathbb{E}\left[P\left(E \mid \hat{\mathbf{h}}_{d}\right)\right]$, can then be expressed as

$$
P(E)=\frac{1}{\pi} \int_{0}^{\pi-\frac{\pi}{M}} \prod_{n=1}^{N_{c}}\left[1+\frac{d_{n} \sin ^{2}\left(\frac{\pi}{M}\right)}{\sin ^{2}(\phi)}\right]^{-1} d \phi,
$$

which can be easily evaluated by numerical calculation.

In case of perfect CSI, we have the assumption $\mathbf{R}_{e e}=\mathbf{0}$ so that the decision variable in (19) simplifies to $\alpha=$ $\frac{1}{\sigma_{z}^{2}} \sqrt{\frac{E_{s}}{N_{s}}} \mathbf{h}_{d}^{H} \mathbf{y}_{d}$. Thus, the optimum decision rule in Proposition (20) degrades to the conventional maximal ratio combining (MRC) receiver. From (26), the unconditional SER simplifies to

$$
P(E)=\frac{1}{\pi} \int_{0}^{\pi-\frac{\pi}{M}} \prod_{n=1}^{N_{c}}\left[1+\frac{g_{n} \sin ^{2}\left(\frac{\pi}{M}\right)}{\sin ^{2}(\phi)} \frac{\gamma_{c}}{N_{c}}\right]^{-1} d \phi,
$$

where $g_{n}$ is the eigenvalue of $\mathbf{R}_{d d}$, and it is directly related to the Doppler diversity gain.

However, in case of imperfect CSI, the result in (20) shows that MRC is no longer optimal. The presence chan- 
nel estimation error affects the decision process, and the new optimum decision rule has to take into consideration the statistical properties of the channel estimation error quantified in the matrix $\mathbf{R}_{e e}$.

The SER expressions in (26) reveal the trade-off between Doppler diversity and channel estimation errors, and they provide a lower bound on the performance of systems with practical precoders.

\section{Doppler domain multiplexing in the presence of imperfect CSI}

The design and performance of a practical rate 1 DDM precoding scheme is studied in this section. A suboptimum receiver is developed by studying the statistical properties of the estimated channel coefficients, and the corresponding analytical error performance is derived.

\subsection{Doppler domain equalization with imperfect CSI}

From (2) and (6), the received coded data samples can be expressed as

$$
\mathbf{y}_{d}=\sqrt{E_{c}} \hat{\mathbf{H}}_{d} \Theta \mathbf{s}+\sqrt{E_{c}} \mathbf{E}_{d} \Theta \mathbf{s}+\mathbf{z}_{d},
$$

where $\hat{\mathbf{H}}_{d}=\operatorname{diag}\left(\hat{\mathbf{h}}_{d}\right)$ and $\mathbf{E}_{d}=\operatorname{diag}\left(\mathbf{e}_{d}\right)$ are diagonal matrices with $\hat{\mathbf{h}}_{d}$ and $\mathbf{e}_{d}$ on their main diagonals, respectively.

The system can be considered as an equivalent MIMO system with the equivalent channel matrix being $\hat{\mathbf{H}}_{d} \Theta$, which introduces interference among the symbols in the frequency domain. In addition, interference is introduced by the channel estimation error.

We propose to detect the transmitted symbols by using an iterative soft-input soft-output (SISO) block decision feedback equalizer (BDFE) [14]. Unlike conventional $\mathrm{BDFE}$ that assume perfect $\mathrm{CSI}$ at the receiver, the BDFE in this paper is developed by considering the statistical properties of the estimated channel coefficients and the channel estimation errors.

The input to the SISO-BDFE equalizer is the a priori log-likelihood ratio (LLR) of the information bits, and the output of the SISO-BDFE equalizer is the a posteriori LLR of the information bits. The a priori LLR at the $i$-th iteration is the a posteriori LLR at the $(i-1)$-th iteration. Based on the a priori LLR of the bits, the a priori mean, $\bar{s}_{n}$, and variance, $\sigma_{n}^{2}$, of the symbol $s_{n}$ can be calculated as described in [14].

The structure of the SISO-BDFE in one iteration is shown in Fig. 1. The BDFE contains two filters, a feedforward filter $\mathbf{A}_{n}$ and a feedback filter $\mathbf{B}_{n}$, both will be calculated by using the properties of $\hat{\mathbf{H}}_{d}$ and $\mathbf{E}_{d}$.

The output of the feedforward filter is $\mathbf{r}=\mathbf{A}_{n}$ $\left(\mathbf{y}_{d}-\hat{\mathbf{H}}_{d} \overline{\mathbf{s}}_{n}\right)$, where $\overline{\mathbf{s}}_{n}=\left[\bar{s}_{1}, \ldots, \bar{s}_{n-1}, 0, \bar{s}_{n+1}, \ldots, \bar{s}_{N_{c}}\right]^{T} \in$ $\mathcal{C}^{N_{c} \times 1}$ is the a priori mean vector. The $n$-th element of $\overline{\mathbf{s}}_{n}$ is set to 0 during the detection of $s_{n}$ to avoid instability caused by positive feedback. The output of the feedforward filter is then used to subtract the output of the feedback filter, $\mathbf{B}_{n}\left(\hat{\mathbf{s}}-\overline{\mathbf{s}}_{n}\right)$, where $\mathbf{B}_{n}$ is a strict upper triangular matrix with the main diagonal being $0, \hat{\mathbf{s}}$ is the hard decision at the output of the BDFE, and the soft output of the BDFE filter, $\tilde{\mathbf{s}}_{n}$, can then be represented by

$$
\tilde{\mathbf{s}}_{n}=\mathbf{A}_{n}\left(\mathbf{y}_{d}-\hat{\mathbf{H}}_{d} \overline{\mathbf{s}}_{n}\right)-\mathbf{B}_{n}\left(\hat{\mathbf{s}}-\overline{\mathbf{s}}_{n}\right)+\overline{\mathbf{s}}_{n},
$$

where the soft output $\tilde{\mathbf{s}}_{n}$ is used for the detection of $s_{n}$.

Since $\mathbf{B}_{n}$ is strictly upper triangular, the detection is performed in a reverse order, that is, $s_{n+1}$ is detected before $s_{n}$ and the hard decision of $s_{n+1}$ is fed back to facilitate the detection of $s_{k}$ for $k<n+1$.

Based on the common assumption of correct past decisions, the error vector $\epsilon_{n}=\tilde{\mathbf{s}}_{n}-\mathbf{s}$ can be written as

$$
\epsilon_{n}=\mathbf{A}_{n}\left(\mathbf{y}_{d}-\hat{\mathbf{H}}_{d} \overline{\mathbf{s}}_{n}\right)-\left(\mathbf{B}_{n}+\mathbf{I}_{N_{c}}\right)\left(\mathbf{s}-\overline{\mathbf{s}}_{n}\right) .
$$

With the orthogonality principle, $\mathbb{E}\left[\epsilon_{n} \mathbf{y}_{d}^{H}\right]=0$, the feedforward matrix $\mathbf{A}_{n}$ can be derived as

$$
\mathbf{A}_{n}=\left(\mathbf{B}_{n}+\mathbf{I}_{N_{c}}\right) \mathbf{R}_{s y} \mathbf{R}_{y y}^{-1}=\left(\mathbf{B}_{n}+\mathbf{I}_{N_{c}}\right) \mathbf{G}_{\mathrm{mmse}}
$$

where $\mathbf{G}_{\text {mmse }}=\mathbf{R}_{s y} \mathbf{R}_{y y}^{-1}$ is the linear MMSE matrix for estimating $\mathbf{s}$ from $\mathbf{y}_{d}, \mathbf{R}_{s y}=\mathbb{E}\left[\left(\mathbf{s}-\overline{\mathbf{s}}_{n}\right)\left(\mathbf{y}_{d}-\hat{\mathbf{H}}_{d} \overline{\mathbf{s}}_{n}\right)^{H}\right]$, and $\mathbf{R}_{y y}=\mathbb{E}\left[\left(\mathbf{y}_{d}-\hat{\mathbf{H}}_{d} \overline{\mathbf{s}}_{n}\right)\left(\mathbf{y}_{d}-\hat{\mathbf{H}}_{d} \overline{\mathbf{s}}_{n}\right)^{H}\right]$. From (27), we have

$$
\begin{aligned}
& \mathbf{R}_{s y}=\sqrt{E_{c}} \mathbf{R}_{s s}\left(\hat{\mathbf{H}}_{d} \Theta\right)^{H}, \\
& \mathbf{R}_{y y}=E_{c}\left(\hat{\mathbf{H}}_{d} \Theta\right) \mathbf{R}_{s s}\left(\hat{\mathbf{H}}_{d} \Theta\right)^{H}+E_{c} \mathbb{E}(\mathbf{T})+\sigma_{z}^{2} \mathbf{I}_{N_{c}},
\end{aligned}
$$

where $\mathbf{R}_{s s}=\operatorname{diag}\left[\sigma_{1}^{2}, \cdots, \sigma_{N_{c}}^{2}\right]$ with $\sigma_{n}^{2}$ being the a priori variance of $s_{n}, \mathbf{T}=\mathbf{E}_{d}\left(\Theta \mathbf{R}_{s s} \Theta^{H}\right) \mathbf{E}_{d}^{H}$. Since $\mathbf{E}_{d}$ is diagonal, the $(m, n)$-th element of $\mathbf{T}$ is $(\mathbf{T})_{m, n}=e_{m} e_{n}^{*} \bar{v}_{m n}$, where $e_{m}$ is the $m$-th element of the channel estimation error vector $\mathbf{e}$, and $\bar{v}_{m n}$ is the $(m, n)$-th element of the matrix $\overline{\mathbf{V}}=$ $\Theta \mathbf{R}_{s s} \Theta^{H}$. Thus $\mathbb{E}\left[(\mathbf{T})_{m, n}\right]=\left(\mathbf{R}_{e e}\right)_{m n} \cdot v_{m n}$. Therefore, the matrix $\mathbb{E}(\mathbf{T})=\mathbf{R}_{e e} \odot \overline{\mathbf{V}}$, where $(\overline{\mathbf{A}} \odot \overline{\mathbf{B}})_{m, n}=(\overline{\mathbf{A}})_{m, n}(\overline{\mathbf{B}})_{m, n}$ is the element-wise multiplication between two matrices.

Combining (29) and (30) yields $\epsilon_{n}=\left(\mathbf{B}_{n}+\mathbf{I}_{N_{c}}\right) \xi_{n}$, where $\xi_{n}=\mathbf{R}_{s y} \mathbf{R}_{y y}^{-1}\left(\mathbf{y}_{d}-\hat{\mathbf{H}}_{d} \overline{\mathbf{s}}_{n}\right)-\left(\mathbf{s}-\overline{\mathbf{s}}_{n}\right)$ is the error vector of a linear MMSE equalizer. Based on the orthogonal principle $\mathbb{E}\left(\xi_{n} \mathbf{y}_{d}^{H}\right)=0$, we have $\Phi_{\xi \xi}=\mathbb{E}\left(\xi_{n} \xi_{n}^{H}\right)=\mathbf{R}_{s s}^{n}-\mathbf{R}_{s y} \mathbf{R}_{y y}^{-1} \mathbf{R}_{s y}^{H}$. Combining (31), (32), and the matrix inverse lemma, we have

$$
\Phi_{\xi \xi}=\left[\left(\mathbf{R}_{s S}\right)^{-1}+\Theta^{H} \hat{\mathbf{H}}_{d}^{H}\left(\mathbf{R}_{e e} \odot \overline{\mathbf{V}}+\frac{1}{\gamma_{c}} \mathbf{I}_{N_{c}}\right)^{-1} \hat{\mathbf{H}}_{d} \Theta\right]^{-1} .
$$




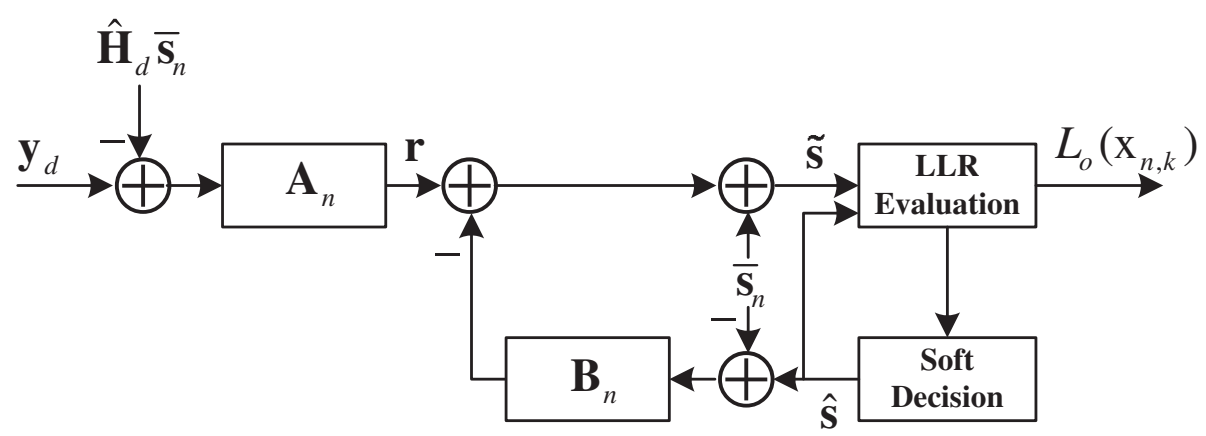

Fig. 1 The soft-input soft-output block decision feedback equalizer

The covariance matrix of the BDFE error vector $\epsilon_{n}$ can then be calculated as $\Phi_{\epsilon \epsilon}=\left(\mathbf{B}_{n}+\mathbf{I}_{N_{c}}\right) \Phi_{\xi \xi}\left(\mathbf{B}_{n}+\mathbf{I}_{N_{c}}\right)^{H}$. We need to identify the feedback matrix $\mathbf{B}_{n}$ such that $\operatorname{trace}\left(\Phi_{\xi \xi}\right)$ is minimized under the constraint that $\mathbf{B}_{n}$ is strictly upper diagonal. The problem can be solved through the Cholesky decomposition of $\Phi_{\xi \xi}^{-1}$ as [15]

$$
\Phi_{\xi \xi}^{-1}=\mathbf{L}^{H} \mathbf{L}=\mathbf{U}_{\xi}^{H} \mathbf{D}_{\xi} \mathbf{U}_{\xi},
$$

where $\mathbf{U}_{\xi} \in \mathcal{C}^{N_{c} \times N_{c}}$ is an upper triangular matrix with unit diagonal elements, $\mathbf{D}_{\xi} \in \mathcal{R}^{N_{c} \times N_{c}}$ is a diagonal matrix, and $\mathbf{L}=\sqrt{\mathbf{D}_{\xi}} \mathbf{U}_{\xi}$. With the Cholesky decomposition described in (33), the feedback matrix $\mathbf{B}_{n}$ can be calculated as $\mathbf{B}_{n}=$ $\mathbf{U}_{\xi}-\mathbf{I}_{N_{c}}$. Consequently, the error covariance matrix of the BDFE equalizer is $\Phi_{\epsilon \epsilon}=\mathbf{D}_{\xi}^{-1}$.

\subsection{Error performance analysis}

The pairwise error probability (PEP) and a BER lower bound of the rate 1 DDM system with imperfect CSI is developed in this subsection.

Based on the estimated channel coefficients, (6) can be rewritten as

$$
\mathbf{y}_{d}=\sqrt{E_{c}} \mathbf{X}_{d} \hat{\mathbf{h}}_{d}+\mathbf{w}_{d}
$$

where $\mathbf{w}_{d}=\sqrt{E_{c}} \mathbf{X}_{d} \mathbf{e}_{d}+\mathbf{z}_{d}$ is the equivalent noise component associated with the estimation error and AWGN. If we treat $\mathbf{w}_{d}$ as noise, then the maximum likelihood rule for deciding between two possible codewords for MPSK modulated systems, $\mathbf{c}_{\alpha}$ and $\mathbf{c}_{\beta}$, is

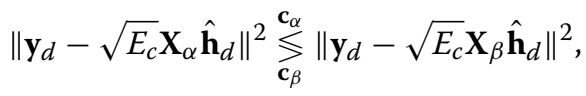

where $\mathbf{X}_{\alpha}=\operatorname{diag}\left(\mathbf{c}_{\alpha}\right)$ and $\mathbf{X}_{\beta}=\operatorname{diag}\left(\mathbf{c}_{\beta}\right)$.

Based on the ML decision rule in (35), the PEP of deciding in favor of $\mathbf{X}_{\beta}$ when $\mathbf{X}_{\alpha}$ is transmitted can be calculated as

$$
P\left(\mathbf{X}_{\alpha} \rightarrow \mathbf{X}_{\beta}\right)=P\left(Q_{z}<0 \mid \mathbf{X}_{\alpha} \text { sent }\right),
$$

where $Q_{z}=\left\|\mathbf{y}_{d}-\sqrt{E_{s}} \mathbf{X}_{\beta} \hat{\mathbf{h}}_{d}\right\|^{2}-\left\|\mathbf{y}_{d}-\sqrt{E_{s}} \mathbf{X}_{\alpha} \hat{\mathbf{h}}_{d}\right\|^{2}$.
Define $\mathbf{z}_{1}=\sqrt{E_{s}}\left(\mathbf{X}_{\alpha}-\mathbf{X}_{\beta}\right) \hat{\mathbf{h}}_{d}+\mathbf{w}_{\alpha}$ and $\mathbf{z}_{2}=\mathbf{w}_{\alpha}$, where $\mathbf{w}_{\alpha}=\sqrt{E_{s}} \mathbf{X}_{\alpha} \mathbf{e}_{d}+\mathbf{z}_{d}$. Conditioned on $\mathbf{X}_{\alpha}$ and $\mathbf{X}_{\beta}$, both $\mathbf{z}_{1}$ and $\mathbf{z}_{2}$ are complex Gaussian distributed. Then $Q_{z}$ can be expressed in a quadratic form of a complex Gaussian random vector as

$$
Q_{z}=\left[\mathbf{z}_{1}^{H} \mathbf{z}_{2}^{H}\right]\left[\begin{array}{cc}
\mathbf{I}_{N_{c}} & \mathbf{0} \\
\mathbf{0} & -\mathbf{I}_{N_{c}}
\end{array}\right]\left[\begin{array}{l}
\mathbf{z}_{1} \\
\mathbf{z}_{2}
\end{array}\right] .
$$

The characteristic function (CHF) of $Q_{z}$ is given by [13]

$$
\Psi_{Q_{z}}(t)=\frac{1}{\operatorname{det}\left(\mathbf{I}_{2 N_{c}}-j t \mathbf{W}_{z}\right)},
$$

where $\mathbf{W}_{z}$ has the form

$$
\mathbf{W}_{z}=\left[\begin{array}{ll}
\mathbf{R}_{z_{1}} & \mathbf{R}_{w_{\alpha}} \\
\mathbf{R}_{w_{\alpha}} & \mathbf{R}_{w_{\alpha}}
\end{array}\right]\left[\begin{array}{cc}
\mathbf{I}_{N_{c}} & \mathbf{0} \\
\mathbf{0} & -\mathbf{I}_{N_{c}}
\end{array}\right]=\mathbf{R}_{z} \cdot \mathbf{K},
$$

with $\mathbf{R}_{z_{1}}=E_{s}\left(\mathbf{X}_{\alpha}-\mathbf{X}_{\beta}\right) \mathbf{R}_{\hat{d} \hat{d}}\left(\mathbf{X}_{\alpha}-\mathbf{X}_{\beta}\right)^{H}+\mathbf{R}_{w_{\alpha}}, \mathbf{R}_{w_{\alpha}}=$ $\mathbb{E}\left[\mathbf{w}_{\alpha} \mathbf{w}_{\alpha}^{H}\right]=E_{s} \mathbf{X}_{\alpha} \mathbf{R}_{e e} \mathbf{X}_{\alpha}^{H}+\sigma_{z}^{2} \mathbf{I}_{N_{c}}$.

Performing eigenvalue decomposition of $\mathbf{R}_{z}$ yields $\mathbf{R}_{z}=$ $\mathbf{U}_{z} \mathbf{D}_{z} \mathbf{U}_{z}^{H}$. The CHF in (37) can be alternatively represented by

$$
\begin{aligned}
\Psi_{Q_{z}}(t) & =\frac{1}{\operatorname{det}\left(\mathbf{I}_{2 N_{c}}-j t \mathbf{D}_{z}^{\frac{1}{2}} \mathbf{U}_{z}^{H} \mathbf{K} \mathbf{U}_{z} \mathbf{D}_{z}^{\frac{1}{2}}\right)} \\
& =\prod_{i=1}^{N_{w}} \frac{1}{1-j t \lambda_{i}},
\end{aligned}
$$

where $\lambda_{i}$ is the $i$-th non-zero eigenvalue of the rank- $N_{w}$ matrix $\mathbf{D}_{z}^{\frac{1}{2}} \mathbf{U}_{z}^{H} \mathbf{K} \mathbf{U}_{z} \mathbf{D}_{z}^{\frac{1}{2}}$.

When the values of $\lambda_{i}$ are all unique, which is usually the case for practical systems, we can rewrite the CHF through partial fraction expansion as

$$
\Psi_{Q_{z}}(t)=\sum_{i=1}^{N_{w}} \frac{\mu_{i}}{1-j t \lambda_{i}},
$$

where $\mu_{i}=\prod_{n=1, n \neq i}^{N_{w}} \frac{\lambda_{i}}{\lambda_{i}-\lambda_{n}}$ 
The PDF of $Q_{z}$ can be derived from the CHF as $f_{Q_{z}}(x)=$ $\frac{1}{2 \pi} \int_{-\infty}^{\infty} \Psi_{Q_{z}}(t) e^{-j x t} d t$, and the result is

$$
f_{Q_{z}}(x)=\sum_{i=1}^{N_{w}} \mu_{i} \cdot \frac{1}{\left|\lambda_{i}\right|} \exp \left(-\frac{x}{\lambda_{i}}\right) u\left(\operatorname{sgn}\left(\lambda_{i}\right) x\right),
$$

where $u(x)$ is the unit step function, and $\operatorname{sgn}(x)=1$ if $x \geq 0$ and -1 otherwise.

Without loss of generality, assume the eigenvalues are ordered in an ascending order, and there are $N_{w}^{-}$negative eigenvalues, the PEP can be written as

$$
P\left(\mathbf{X}_{\alpha} \rightarrow \mathbf{X}_{\beta}\right)=\int_{-\infty}^{0} f_{Q_{z}}(x) d x=\sum_{n=1}^{N_{w}^{-}} \prod_{i=1, i \neq n}^{N_{w}} \frac{\lambda_{n}}{\lambda_{n}-\lambda_{i}}
$$

where $\lambda_{n}$ for $n=1, \ldots, N_{w}^{-}$are the negative eigenvalues.

For a pair of information vectors, $\mathbf{s}_{\alpha}$ and $\mathbf{s}_{\beta}$, their Hamming distance are defined as $D_{H}\left(\mathbf{s}_{\alpha}, \mathbf{s}_{\beta}\right)=\left\|\mathbf{s}_{\alpha}-\mathbf{s}_{\beta}\right\|_{0}$, where $\|\mathbf{a}\|_{0}$ is the $l_{0}$ norm operator that returns the number of non-zero elements in the vector a. Intuitively, the BER can be reduced by assigning codeword pairs with smaller PEP to information vector pairs with larger Hamming distance, such that the probability of error events with a large number of bit errors is small. Equivalently, when $D_{H}\left(\mathbf{s}_{\alpha}, \mathbf{s}_{\beta}\right)$ is small, a good precoding scheme should yield a relatively large PEP $P\left(\mathbf{X}_{\alpha} \rightarrow \mathbf{X}_{\beta}\right)$, where $\mathbf{X}_{\alpha}$ and $\mathbf{X}_{\beta}$ are the codewords of $\mathbf{s}_{\alpha}$ and $\mathbf{s}_{\beta}$, respectively.

Figure 2 shows the PEP of the DDM-precoded system with BPSK modulation and different Hamming dis- tances between information vector pairs. The normalized Doppler frequency is $f_{\mathrm{D}} T_{s}=0.01$ and block length is 100 . For a given Hamming distance $D_{H}$, we randomly generate an information vector $\mathbf{s}_{\alpha}$, then randomly change $D_{H}$ bits in $\mathbf{s}_{\alpha}$ to get $\mathbf{s}_{\beta}$. The PEP for a given Hamming distance is obtained by averaging over $10^{5}$ such information vector pairs. It can be seen that the PEP decreases as $D_{H}\left(\mathbf{s}_{\alpha}, \mathbf{s}_{\beta}\right)$ increases, and the system performance is dominated by error events with 1 bit information error.

Since the system performance is dominated by error events with 1 bit information error, we can get a BER lower bound of a BPSK modulated system by considering only error events with 1 bit error

$$
P_{b} \leq \frac{1}{2^{N_{c}}} \sum_{\alpha=1}^{2^{N_{c}}} \sum_{\substack{\beta: \\ D_{H}\left(\mathbf{s}_{\alpha}, \mathbf{s}_{\beta}\right)=1}} \frac{1}{N_{c}} P\left(\mathbf{X}_{\alpha} \rightarrow \mathbf{X}_{\beta}\right) .
$$

In the equation above, the outer summation with respect to $\alpha$ is used to average over all $2^{N_{c}}$ possible values of $\mathbf{s}_{\alpha}$. When $N_{c}$ is large, the averaging operation is time-consuming. However, it can be evaluated by using a large number of randomly generated $\mathbf{s}_{\alpha}$ instead. It should be noted that our analysis can be easily extended to any MPSK modulated system by considering the dominant error events.

\section{Simulation results}

Analytical and simulation results are presented in this section to study the trade-off between Doppler diversity

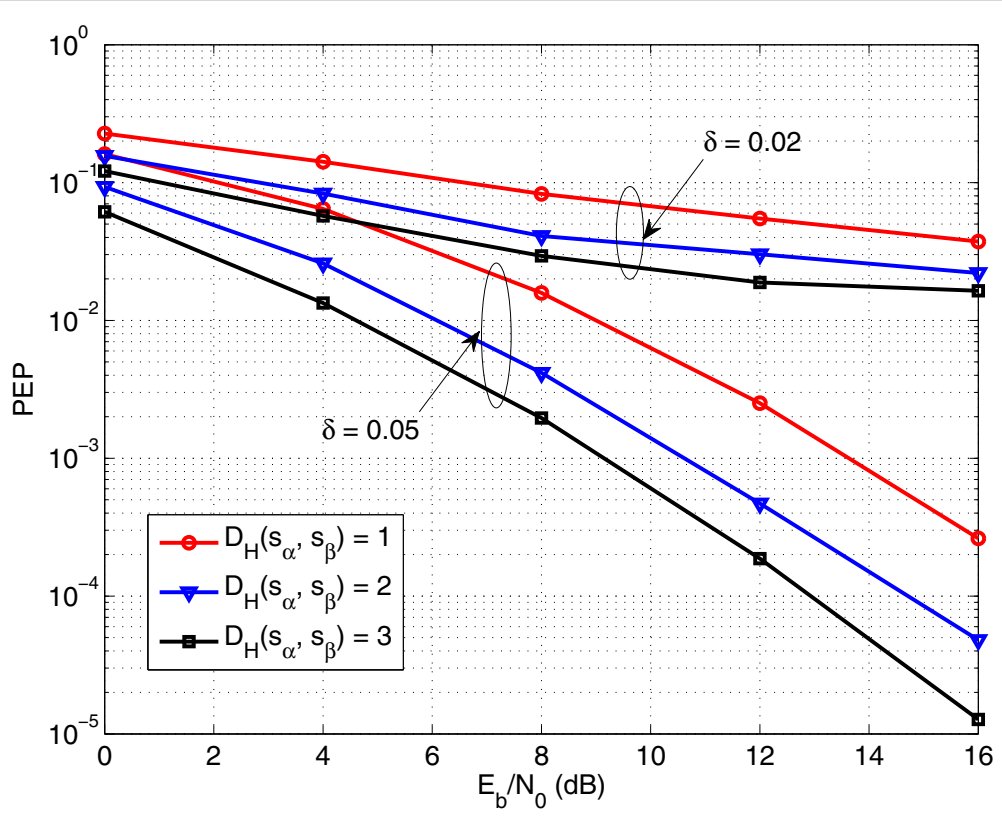

Fig. 2 The PEP as a function of $E_{b} / N_{0}$ for IDFT precoding systems with imperfect channel state information 
and channel estimation errors and to validate the performance of the two precoding schemes in the presence of imperfect CSI. All systems employ a symbol rate of 0.1 Msym/s and operating at $1.9 \mathrm{GHz}$. The block length is $N=100$. When the movement speed is between 56.8 and $568.4 \mathrm{~km} / \mathrm{hr}$, the corresponding range of Doppler spread is between $100 \mathrm{~Hz}\left(f_{\mathrm{D}} T_{s}=10^{-3}\right)$ to $1 \mathrm{KHz}\left(f_{\mathrm{D}} T_{s}=10^{-2}\right)$.

Figure 3 compares the performance between the two precoding schemes with both analytical and simulation results. For the rate $\frac{1}{N_{c}}$ repetition coded system, the analytical BER described in (26) serves as the lower bound for Doppler diversity system in the presence of channel estimation error, while the simulated BER is obtained by utilizing the optimum receiver described in (20). The rate 1 DDM system employs the suboptimum SISO-BDFE receiver and the analytical results are obtained by using (44). The value of $f_{\mathrm{D}} T_{s}$ is 0.01 , and the energy allocation factor is selected as $\eta=0.1$. The analytical BER of the rate 1 system matches very well with the corresponding simulation results. The analytical BER lower bound of the DDM-precoded system can reasonably predict the performance of the system with the sub-optimum SISO-BDFE receivers. For both systems, the pilot percentage has significant impacts on the BER performance. When $\delta=0.02$, the BER performance is dominated by channel estimation errors, such that the Doppler diversity gain is very small. When $\delta=0.05$, the repetition coded system can achieve the maximum Doppler diversity order as the system with perfect CSI. The BER of the DDM precoded system is 1.9 $\mathrm{dB}$ worse than the repetition coded system at $10^{-3}$ and $\delta=0.05$, mainly due to the interference introduced by the rate 1 precoder. Similar performance is observed for high-level modulations.

Similar results are given in Fig. 4 with higher modulation order. For the rate $\frac{1}{N_{c}}$ repetition code, the analytical BER is approximated by dividing the SER over $\log _{2}(M)$, and a perfect match is observed between the analytical and simulation SER. For the rate 1 DDM scheme, the analytical results serve as the lower bound for the simulation results.

Figure 5 shows the analytical and simulated BER as a function of the energy allocation factor $\eta$ for system with the repetition precoding scheme. The normalized Doppler spread is $f_{\mathrm{D}} T_{s}=0.01$ and $E_{b} / N_{0}=16 \mathrm{~dB}$. Simulation and analytical results match very well. For all system configurations, the BER is convex in $\eta$. The optimum $\eta$ increases as $\delta$ increases. The optimum $\eta$ is $0.06,0.09$, $0.1,0.1$ and 0.1 for systems with $\delta=0.01,0.02,0.05,0.1$, and 0.5 , respectively. A larger $\delta$ means more pilot symbols per slot; thus, more energy needs to be allocated to the $N_{p}$ pilot symbols in order to achieve a good channel estimation quality. Similar results are also observed for systems with DDM precoders. We will use the energy allocation factor 0.1 for all subsequent examples, and it provides a robust and good performance for all system configurations.

Figure 6 shows the analytical BER lower bound as a function of pilot percentage, under various values of $f_{\mathrm{D}} T_{s}$. The energy allocation factor is $\eta=0.1$ and $E_{b} / N_{0}=16$ $\mathrm{dB}$. When $\delta$ is small, e.g., $\delta<0.05$ for $f_{\mathrm{D}} T_{s}=0.01$, increasing $\delta$ slightly yields significant performance gains because the performance is dominated by channel estimation errors. Once $\delta$ reaches a threshold, e.g., $\delta \geq 0.1$ for

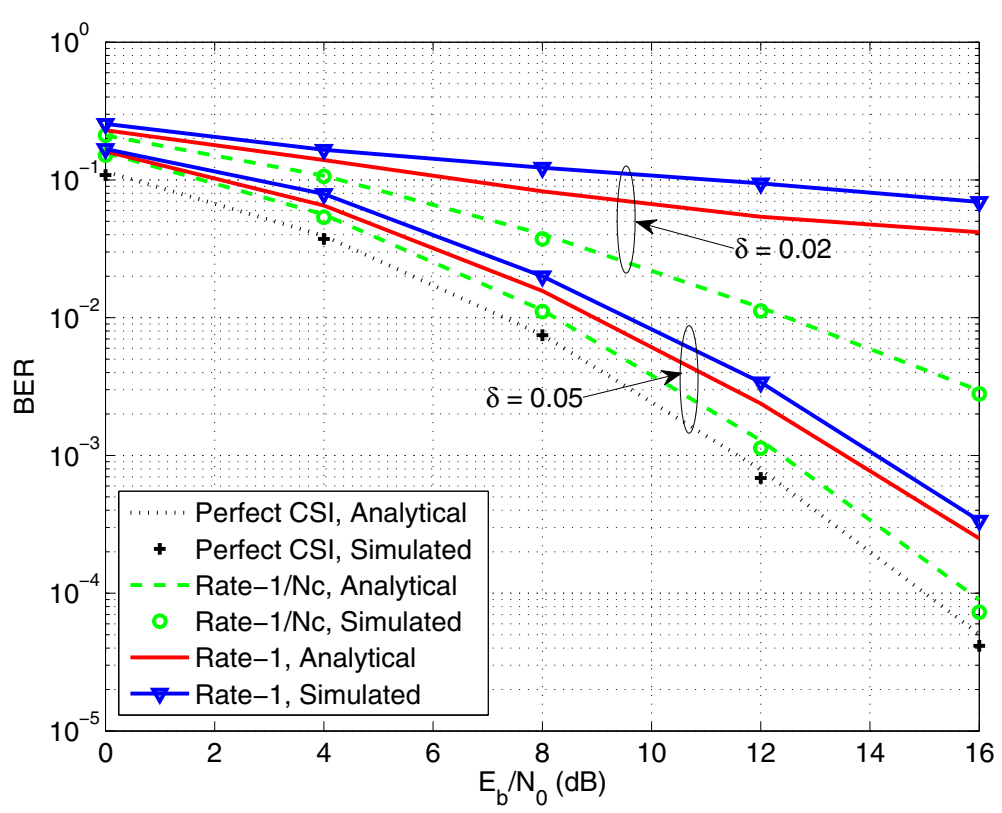

Fig. 3 The analytical and simulation results for both rate $\frac{1}{N_{c}}$ and rate 1 diversity systems with BPSK and imperfect channel state information 


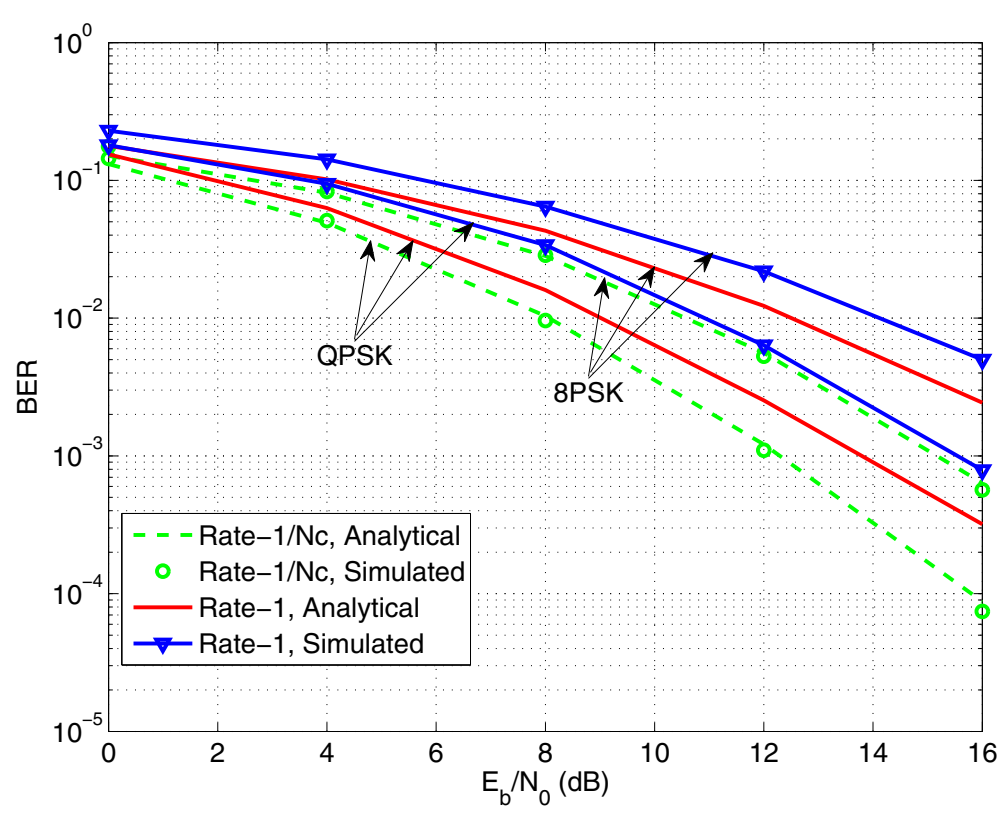

Fig. 4 The analytical and simulation results for both rate $\frac{1}{N_{c}}$ and rate 1 diversity systems with QPSK/8PSK and imperfect channel state information

$f_{\mathrm{D}} T_{s}=0.01$, increasing $\delta$ further has very small impact on system performance, due to the fact that the impacts of channel estimation error is small once $\delta$ becomes large. It is interesting to note that increasing $\delta$ from 0.1 to 0.5 might degrade system performance because $E_{p}$ is a decreasing function in $\delta$. More importantly, when $\delta$ is small (e.g. $\delta<0.02$ ), a higher $f_{\mathrm{D}} T_{s}$ might result in a worse BER due to channel estimation errors. On the other hand, when $\delta$ is large enough such that the channel estimation quality is good enough, the BER performance increases with $f_{\mathrm{D}} T_{s}$ due to the higher Doppler diversity gains collected by the proposed systems.

To illustrate the impact of Doppler shift on error performance, the BER of system with repetition code is shown as a function of $f_{\mathrm{D}} T_{s}$ in Fig. 7, under various values of pilot percentage. The $E_{b} / N_{0}$ is fixed at $16 \mathrm{~dB}$. For all

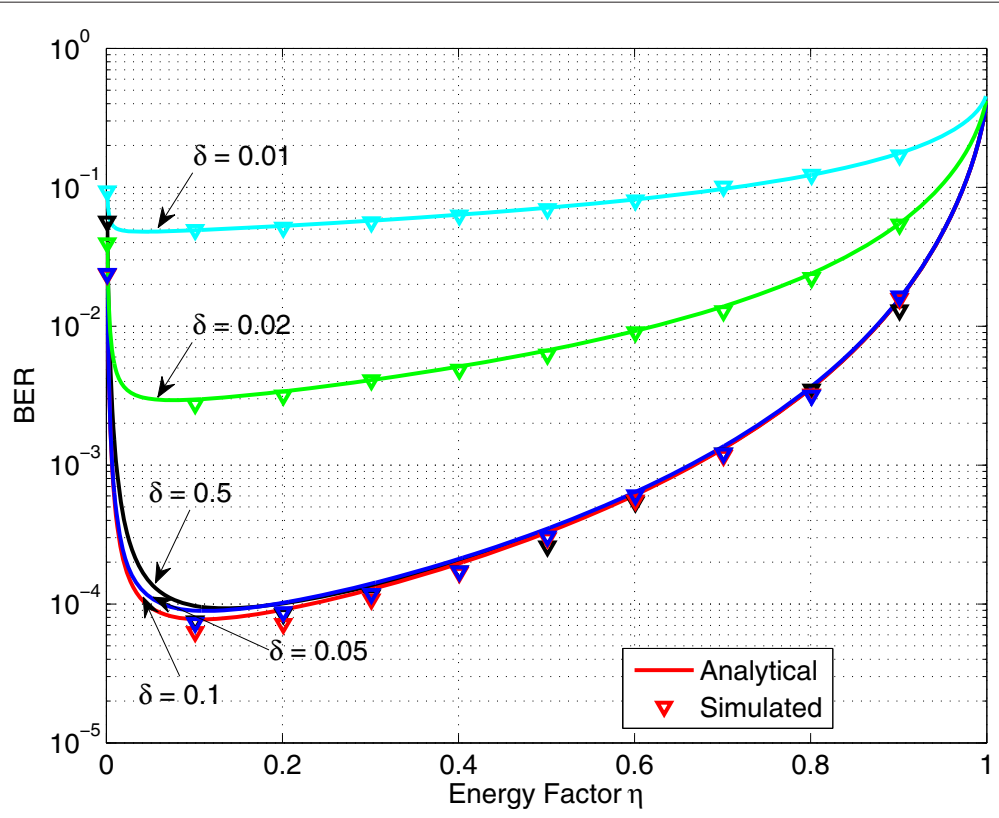

Fig. 5 The BER as a function of $\eta$ for rate $\frac{1}{N_{c}}$ system with BPSK modulation and imperfect channel state information 


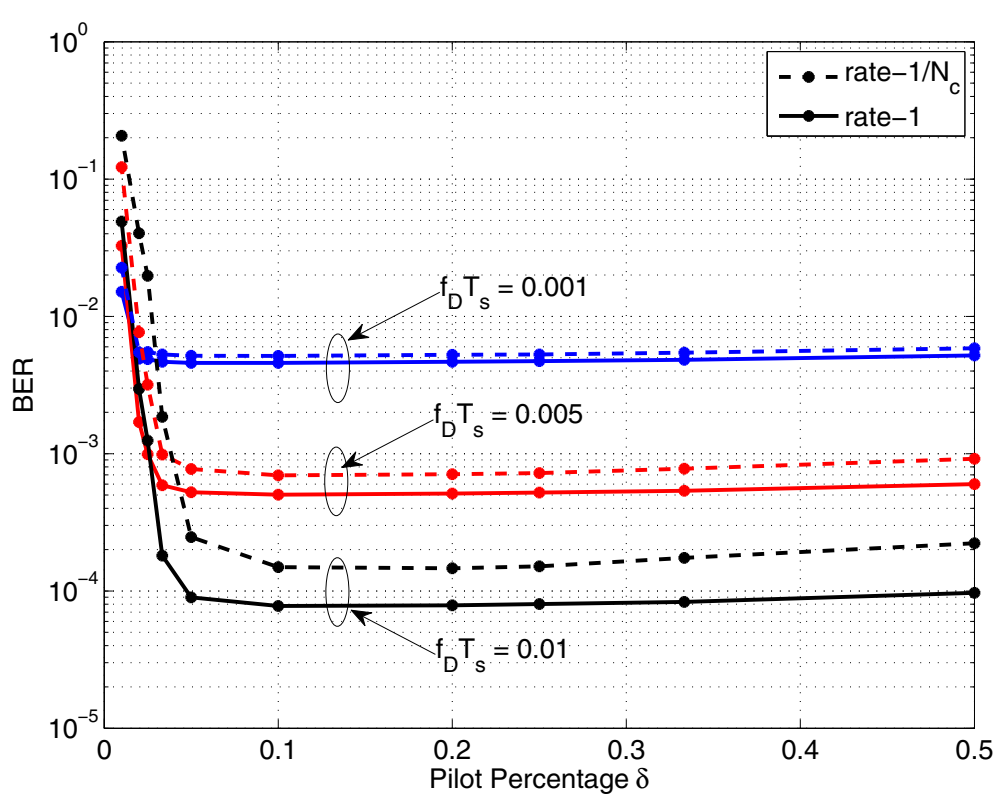

Fig. 6 The BER as a function of $\delta$ for rate $\frac{1}{N_{c}}$ systems with BPSK modulation and imperfect channel state information

systems, the BER is a quasi-convex function of $f_{\mathrm{D}} T_{s}$. The performance first improves as $f_{\mathrm{D}} T_{s}$ increases due to the Doppler diversity and then degrades due to the channel estimation errors. For systems with $\delta=0.02,0.05$, and 0.1 , the minimum BER is achieved at $f_{\mathrm{D}} T_{s}=0.004,0.015$, and 0.045 , respectively. A higher pilot percentage may not always yield a better performance, and the performance improvement is not significant when the channel estimation is good enough.

\section{Conclusions}

The maximum Doppler diversity transmissions for highmobility systems in the presence of channel estimation errors have been studied in this paper. The trade-off between Doppler diversity and channel estimation errors has been studied by using a repetition code and a rate 1 Doppler domain multiplexing scheme. The analytical performance of both systems have been obtained by analyzing the statistical properties of the channel estimation

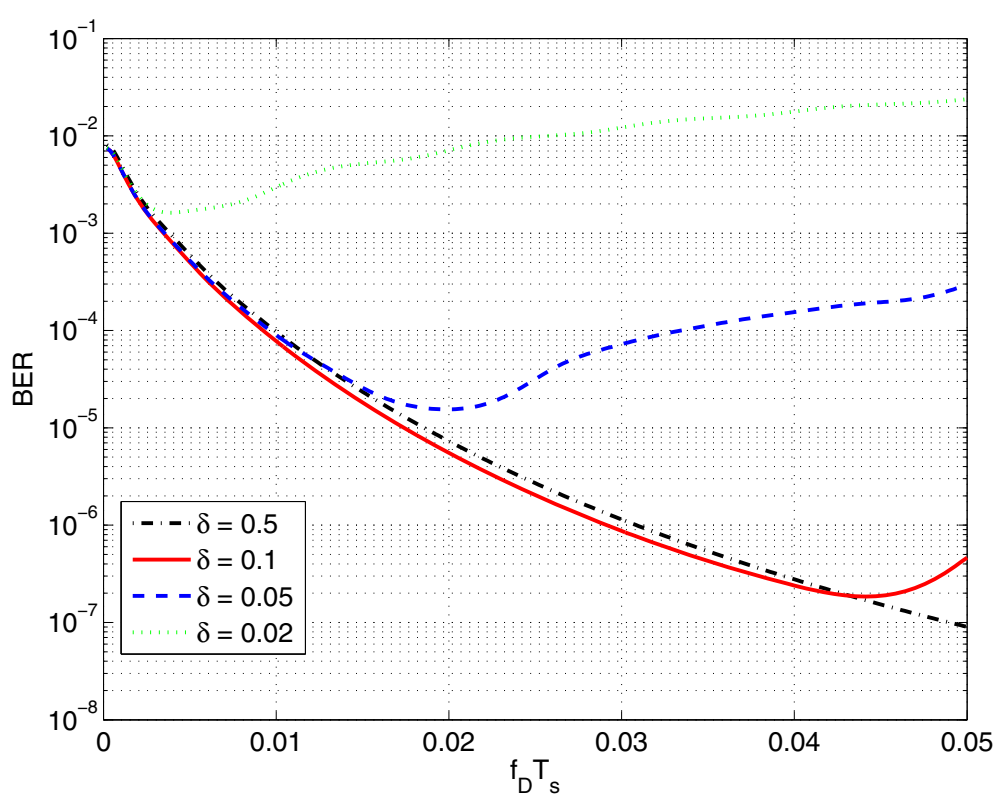

Fig. 7 The BER as a function of $f_{D} T_{s}$ for rate $\frac{1}{N_{c}}$ systems with BPSK modulation and imperfect channel state information 
errors, and they quantitatively identify the impacts and interactions of a number of system parameters, such as the pilot percentage, the maximum Doppler spread, and the energy allocation factor between pilot and data symbols, etc. It has been shown that the error probability is quasiconvex in Doppler spread and monotonically decreasing in the pilot percentage. The performance of systems with a sufficiently high pilot percentage can approach that of a system with perfect CSI. On the other hand, if the pilot percentage is too low, the benefits of Doppler diversity are offset by channel estimation error such that a system with a lower Doppler spread could get a better performance.

\section{Competing interests}

The authors declare that they have no competing interests.

\section{Acknowledgements}

The work of Weixi Zhou and Pingzhi Fan was supported by the Chinese 973 Program (No. 2012CB316100), the NSFC project (No. 61471302), the 111 Project (No. 111-2-14), the MoE Key Grant Project (No. 311031100), and the Young Innovative Research Team of Sichuan Province (No. 2011JTD0007). The work of Jingxian Wu was supported in part by the U.S. National Science Foundation (NSF) under Grants ECCS-1202075 and ECCS-1405403.

\section{Author details}

${ }^{1}$ Institute of Mobile Communications, Southwest Jiaotong University, Chengdu, China. ${ }^{2}$ Department of Electrical Engineering, University of Arkansas, AR 72701 Fayetteville, USA.

Received: 7 December 2014 Accepted: 29 April 2015

Published online: 21 May 2015

\section{References}

1. AM Sayeed, B Aazhang, Joint multipath-Doppler diversity in mobile wireless communications. IEEE Trans. Commun. 47(1), 123-132 (1999). doi:10.1109/26.747819

2. $\mathrm{X} M \mathrm{Ma}, \mathrm{GB}$ Giannakis, Maximum-diversity transmissions over doubly selective wireless channels. IEEE Trans. Inform. Theory. 49(7), 1832-1840 (2003). doi:10.1109/TIT.2003.813485

3. J Wu, in Proc. IEEE GLOBECOM. Exploring maximum Doppler diversity by Doppler domain multiplexing (San Fransico, USA, 2006), pp. 1-5. doi:10.1109/GLOCOM.2006.897

4. KI Ahmed, C Tepedelenlioglu, A Spanias, Performance of precoded OFDM with channel estimation error. IEEE Trans. Signal Process. 54(3), 1165-1171 (2006). doi:10.1109/TSP.2005.862898

5. J Wu, C Xiao, Optimal diversity combining based on linear estimation of Rician fading channels. IEEE Trans. Commun. 56(10), 1612-1615 (2008). doi:10.1109/TCOMM.2008.060598

6. S Savazzi, U Spagnolini, On the pilot spacing constraints for continuous time-varying fading channels. IEEE Trans. Commun. 57(11), 3209-3213 (2009). doi:10.1109/TCOMM.2009.11.080024

7. N Sun, J Wu, Maximizing spectral efficiency for high mobility systems with imperfect channel state information. IEEE Trans. Wireless Commun. 13(3), 1462-1470 (2013). doi:10.1109/TWC.2014.012314.130772

8. N Sun, J Wu, in Proc. IEEE GLOBECOM. Maximizing spectral efficiency with imperfect channel information in high mobility systems (Atlanta, USA, 2013). doi:10.1109/GLOCOM.2013.6831591

9. W Zhou, J Wu, P Fan, in Proc. IEEE ICC. Maximizing Doppler diversity transmisssions for high mobility systems with imperfect channel state information (Sydney, Australia, 2014). doi:10.1109/ICC.2014.6884267

10. J Wu, N Sun, Optimum sensor density in distortion tolerant wireless sensor networks. IEEE Trans. Wireless Commun. 11, 2056-2064 (2012). doi:10.1109/TWC.2012.041612.110127

11. N Sun, J Wu, Optimum sampling in spatial-temporally correlated wireless sensor networks,. EURASIP J. Wireless Commun. Networking. 2013, 5 (2013). doi:10.1186/1687-1499-2013-5
12. N Sun, J Wu, in Proc. IEEE MILCOM. Minimum error transmissions with imperfect channel information in high mobility systems (San Diego, USA 2013). doi:10.1109/MILCOM.2013.160

13. S Siwamogsatham, MP Fitz, JH Grimm, A new view of performance analysis of transmit diversity schemes in correlated Rayleigh fading. IEEE Trans. Inform. Theory. 48(4), 950-956 (2002). doi:10.1109/18.992794

14. J Wu, YR Zheng, Low complexity soft-input soft-output block decision feedback equalization. IEEE J. Sel. Areas Commun. 26(2), 281-289 (2008) doi:10.1109/JSAC.2008.080205

15. A Stamoulis, GB Giannakis, A Scaglione, Block FIR decision-feedback equalizers for filterbank precoded transmissions with blind channel estimation capabilities. IEEE Trans. Commun. 49(1), 69-83 (2001). doi:10.1109/26.898252

\section{Submit your manuscript to a SpringerOpen ${ }^{\mathcal{O}}$ journal and benefit from:}

- Convenient online submission

- Rigorous peer review

- Immediate publication on acceptance

- Open access: articles freely available online

- High visibility within the field

- Retaining the copyright to your article

Submit your next manuscript at $\boldsymbol{\wedge}$ springeropen.com 